Wimblu, Rev. Estud. de Psic. y Cienc. Soc. UCR, 14(1) 2019 (Enero-Junio): 139-152 /ISSN: 1659-2107

\title{
Memorias del Trabajo Comunal Universitario en Promoción de la Salud Mental Costarricense
}

\author{
Memories of University Community Work in promotion \\ of Costa Rican mental health
}

Ana Lucía Rodríguez Gutiérrez ${ }^{1}$

\begin{abstract}
RESUMEN
En el presente artículo se expone a modo de ensayo las memorias del Trabajo Comunal Universitario realizado durante el año 2018 en promoción de la salud mental costarricense, con el fin de divulgar labores realizadas en espacios de salud como parte de la acción social universitaria (UCR). A modo de introducción, se presenta una revisión del marco teórico que fundamenta la promoción y prevención en salud mental, el contexto del sistema sanitario nacional y la descripción del TCU-505. En el ensayo se presentan las principales ideas sobre el trabajo realizado en los distintos espacios institucionales y extra-institucionales. Finalmente, se concluye a raíz de las labores realizadas la importancia de: (1) Fomentar redes de apoyo en el trabajo sanitario, (2) Llevar a cabo una planificación articulada a las condiciones particulares de la población meta, (3) La amplia demanda nacional en salud mental y (4) La aplicación de un enfoque bio-psico-social (interdisciplinariedad) en miras de promover una salud integral.

Palabras clave: Promoción de la salud, salud mental, salud integral, Trabajo Comunal Universitario, Hospital Psiquiátrico Chacón Paut, Centro Diurno Chacón Paut, Unidad de Cuidados Paliativos, Asociación de Personas Adultas Mayores, Santa María de Dota.
\end{abstract}

\begin{abstract}
In the present article the reports of the University Community Work carried out during the year 2018 in promotion of the Costa Rican mental health, in order to disclose work done in health spaces as part of the university social action (UCR), are exposed as an essay. As an introduction, it presents a review of the theoretical framework that supports mental health promotion and prevention, the context of the national health system and the description of the TCU-505. The essay presents the main ideas about the work carried out in the different institutional and extra-institutional spaces. Finally, it is concluded the importance of: (1) Encouraging support networks in health work, (2) Carrying out an articulated planning to the particular conditions of the target population, (3) The broad demand national in mental health and (4) The application of a bio-psycho-social (interdisciplinary) approach in order to promote integral health.

Palabras clave: Promoción de la salud, salud mental, salud integral, Trabajo Comunal Universitario, Hospital Psiquiátrico Chacón Paut, Centro Diurno Chacón Paut, Unidad de Cuidados Paliativos, Asociación de Personas Adultas Mayores, Santa María de Dota.

Key Words: Health promotion, mental health, integral health, University Community Work, Chacón Paut Psychiatric Hospital, Chacón Paut Day Center, Palliative Care Unit, Senior Association, Santa María de Dota.
\end{abstract}

\footnotetext{
${ }^{1}$ Universidad de Costa Rica. Estudiante de Psicología y Enseñanza de la Psicología. Costa Rica. Correo electrónico: ana.rodriguezgutierrez@ucr.ac.cr

Recepción: 20/12/2018 Aceptación: 2/04/2019
} 


\section{Introducción}

Actualmente, la salud es entendida como un "estado de completo bienestar físico, mental y social, y no solamente la ausencia de afecciones o enfermedades" (Organización Mundial de la Salud 2013:1). Por tanto, hoy día se muestra cada vez más imperiosa la importancia de que los abordajes sanitarios partan desde un modelo bio-psico-social, de modo que se considere la interrelación constante de dichos factores y su impacto en la calidad de vida de las personas, y para lo cual es imprescindible el trabajo interdisciplinario (Brannon, Feist \& Updegraff 2013). Dicho enfoque presenta una asociación relevante al trabajo en prevención y promoción de la salud, ya que constituye el objetivo fundamental de la atención primaria en el sistema sanitario nacional. La importancia de la promoción en salud radica en que facilita la reducción del número de personas con enfermedades crónicas y lesiones, disminuye inequidades sociales que repercuten directamente sobre la salud y la calidad de vida de las personas, y reduce costos por la atención e intervención de padecimientos crónicos que engloba tanto afecciones fisiológicas como psiquiátricas (Sarafino \& Smith 2010, Stephens 2008). En este sentido, el trabajo comunitario se torna fundamental por su dimensión participativa y de amplia cobertura social (Dickinson 2011, Stephens 2008).

En el contexto costarricense, la Caja Costarricense del Seguro Social (CCSS) constituye el sistema de salud que brinda la mayor cobertura poblacional, dado su carácter público y solidario. En sus centros de atención primaria se objetiva proveer servicios de salud a nivel individual, familiar y comunitario, cuya principal meta es la promoción de la salud. Sin embargo, la extensa cobertura poblacional de servicios, en promedio cuatro mil habitantes por centro (Presidencia de la República de Costa Rica 2016), nos presenta una realidad donde precisamente, la mayores necesidades de atención en salud del país provienen de enfermedades crónicas (Organización para la Cooperación y el Desarrollo Económico 2017) y en el caso de salud mental, se aprecia un alto egreso del servicio de psiquiatría (5122 en total para el año 2017, con una concentración mayor en edades de 2044 años) por padecimientos como esquizofrenia, trastornos mentales y del humor, dependencia de alcohol, trastornos mentales no psicóticos y retraso mental (Caja Costarricense del Seguro Social 2017). 
Cabe destacar que el contraste de demanda versus cobertura se visualiza en el último Informe sobre el Sistema de Salud Mental en Costa Rica (IESM-OMS 2008), donde se identifica una ausencia de programas especializados para la atención comunitaria de primer nivel y la insuficiencia de recursos en salud mental, centralizados principalmente en los hospitales psiquiátricos. Dada la situación del país en materia de salud, la recepción de apoyo por parte de instituciones externas al sistema sanitario se muestra crucial para brindar una mayor cobertura poblacional.

Como parte de las experiencias interdisciplinarias que integra actividades universitarias para el aporte solidario a grupos sociales y comunidades vulnerables, el Trabajo Comunal Universitario (TCU) se define como "una actividad de acción social que vincula a grupos y comunidades vulnerables con la población estudiantil que cursa un plan de estudios en la Universidad de Costa Rica, cuyo propósito es contribuir con las transformaciones que la sociedad necesita" (Consejo Universitario 2018:1). Específicamente, el TCU-505 Estrategias para la promoción del mejoramiento de la salud mental busca favorecer en instituciones como el Hospital Psiquiátrico Roberto Chacón Paut, el Centro Diurno del Hospital Chacón Paut, la Unidad de Cuidados Paliativos y la Asociación de Personas Adultas Mayores, mediante la colaboración interdisciplinaria en "programas y actividades que fomenten la rehabilitación, autonomía y autorrealización de los usuarios residentes e internados en estas instituciones, así como apoyar a nivel educativo, reflexivo y analítico, a diversas comunidades que lo requieren” (Jara 2018:1), como tal fue el caso en la feria de salud realizada en Santa María de Dota.

Bajo el objetivo del TCU-505, que engloba alcanzar "a través de la labor estudiantil desde una esfera rehabilitativa, preventiva y promocional, impulsar un mayor conocimiento para favorecer la estabilidad emocional, cognitiva, social, laboral, judicial y espiritual que prevenga al sujeto, la presencia de conductas poco adaptativas ante el enfrentamiento de los problemas cotidianos y perjudiciales para la vida" (Jara 2018:1), se realizaron toda una serie de actividades enfocadas en la prevención y promoción de la salud con personas de diferentes zonas de Costa Rica, a cargo de tres estudiantes (dos futuros profesionales en Enfermería y una futura profesional en Psicología), bajo la tutela de una profesora supervisora y dos asistentes. 
La importancia del trabajo interdisciplinario hoy, se justifica sobre la marcha de la política de salud mental actual donde las actividades en promoción de la salud y prevención primaria toman un rol determinante en espacios comunitarios, de mano con la desinstitucionalización psiquiátrica que se promoverá en un lapso no mayor a tres años (Ministerio de Salud 2012). Específicamente para el trabajo comunal universitario realizado durante el año dos mil dieciocho, las labores dentro de las instituciones psiquiátricas permitieron apoyar a los usuarios desde el departamento de terapia ocupacional para asentar las bases de su comportamiento una vez egresados del hospital, y extrainstitucionalmente, promoviendo estilos de vida saludables con técnicas y estrategias de afrontamiento científicamente válidas a través de una psicoeducación preventiva (Labrador 2009).

El propósito de este artículo es narrar las memorias que surgen a raíz del trabajo de campo realizado, de modo que sea posible divulgar una pequeña parte de la labor que realizan los estudiantes en espacios de salud como parte de la acción social universitaria.

Miércoles en San Ramón de Tres Ríos. Un trayecto de cuarenta minutos permite la visita a montañas de clima nublado, que resguardan en sus adentros el hogar de cuarenta personas. En el Hospital Chacón Paut, los usuarios comienzan su día desde temprano, se despiertan a las seis de la mañana para esperar el desayuno a las siete, mientras caminan libremente por los pasillos. La jornada continúa con distintos programas de terapia ocupacional, donde la lavandería, el cepillado de dientes y la limpieza de las casas toman lugar la primera mitad del día. Barriendo, limpiando muebles y acomodando prendas es como los pacientes de este pabellón refuerzan su funcionalidad y autonomía, cada uno a su propio ritmo.

Bajo cubierta y reforzado con tablones de madera, se encuentra el huerto al lado de las casas. Maíz, rábano, culantro, café son sólo algunos de los cultivos que se pueden apreciar germinados sobre sustrato de fibra de coco. La composta casera es preparada y utilizada por los usuarios para el cuidado de las plantas, gracias a la supervisión del técnico a cargo. Las actividades en huerta preparan a los usuarios, no solamente en el desempeño de la ocupación agrícola; les brinda algo más allá cargado con un sentido físico y emocional. Trabajar con plantas les implica trabajo en equipo, un compartir con otros, tacto 
con la naturaleza y desarrollar sensibilidad hacia el cuidado de otros seres vivos. Tras semanas de esfuerzo, las cosechas son vendidas al personal del mismo Hospital, y los fondos son recaudados para organizar paseos con los usuarios. En el cultivo, cada persona que labora en ese pequeño huerto puede encontrar una compensación que excede la remuneración económica; es la satisfacción de encontrar un objetivo común en lo que se hace, y con ello, una vinculación social al grupo de pertenencia.

La hora para la recreación empieza cerca de las nueve y media de la mañana, bajo la planificación de los estudiantes del TCU. Distintas actividades lúdicas son preparadas con antelación para que todos los usuarios puedan ser partícipes, indistintamente de la variabilidad interindividual de sus condiciones físicas y mentales. Baloncesto, golf, bolos, frisbees, hula-hulas, cometas, relajación, juegos con pelotas son algunas ideas desarrolladas durante el trabajo de campo, cuya planificación era cuidadosamente elaborada por los estudiantes encargados, y posteriormente aprobada por la supervisora a cargo. Cada actividad fue realizada con un propósito específico para la estimulación de sus áreas cognitiva, física y social. Era apreciable en días lluviosos ver cómo los usuarios se acercaban, bajo nuestras sombrillas, a jugar en la explanada bajo techo. En días más soleados, nos aventurábamos en una caminata hacia el terreno norte del Hospital, en cuya cima nos esperaban los pollitos de cemento ${ }^{2}$. Paso a paso llegaba cada quien a su propio ritmo, deseosos de sentarse y degustar la merienda de mitad de día.

Tras una mañana de trabajo común en el Hospital, los usuarios ingresan al comedor para almorzar. El trabajo de los técnicos continúa reforzando la autonomía de los usuarios fomentando el aseo personal, con la responsabilidad de cada quien de colocar los cubiertos sucios en su lugar y procurando que cada usuario lave sus manos al finalizar. Los talleres de terapia ocupacional continúan su jornada en horas de la tarde, donde nuevamente, la facilitación de los estudiantes toma lugar. Desde este espacio, la estimulación cognitiva se lleva a cabo a través de distintas aplicaciones artísticas, lúdicas o audiovisuales. Entre pinturas, música, origami, maquetas, festejos y videos educativos, se reforzó en los usuarios distintas áreas y capacidades; trabajo en equipo, memoria, coordinación visomotora fina y gruesa, atención, creatividad, socialización e incluso dispersión, como recurso indispensable en la promoción de la salud mental.

2 En Costa Rica, "pollitos" es la jerga que hace alusión a una estructura circular de cemento que funciona como asiento colectivo para descansar en espacios al aire libre. 
Durante este trabajo vespertino, era común escuchar el llamado de algún paciente que deseaba ser atendido. El desafío de trabajar con los usuarios cada vez parecía acrecentar por la alta demanda que implicaba la atención individualizada, y en contraposición, la poca cantidad de estudiantes que nos encargábamos de dirigir las actividades. Si bien el apoyo de los técnicos y el trabajo en equipo resultó fundamental en el desarrollo de las actividades, se torna de vital importancia recordar el aprendizaje derivado de este aporte; la gran variabilidad de condiciones y con ella, el empeño y ajuste que implica atender población psiquiátrica. Ante esto, cabe destacar que la literatura de neuropsicología y promoción de la salud sostiene la importancia de conocer las condiciones psicosociales particulares de la población de trabajo, tanto a nivel individual-personal como colectivo-social. De este modo, los objetivos y el trabajo planificado adquieren una orientación acorde a la dimensión poblacional específica (Moore \& Mateer 2001; Wood, Allen \& Pantelis 2009; Lezak et al. 2012; Ardila y Rosselli 2007; Portellano 2005; Wilson et al. 2017; Brannon, Feist \& Updegraff 2013; Sarafino \& Smith 2010; Stephens 2008).

Cabe destacar que a lo largo de los pasillos del Hospital, aún es posible observar en grandes afiches algunos datos sobre la Política Nacional de Salud Mental 2012-2021 de la CCSS (Ministerio de Salud 2012). Aplicada a la realidad inmediata, una de sus principales propuestas para el presente año es llevar a cabo la reubicación de usuarios a centros de cuido para adultos mayores, ya que la gran mayoría de personas institucionalizadas han llegado a una condición de vejez. A partir de esta política, se enfatiza que la institucionalización únicamente responderá a estados de crisis con una permanencia no mayor a la fase aguda, es decir, durante algunas semanas con su posterior reintegración al círculo personal y social.

A tres kilómetros hacia el centro de Tres Ríos, se ubica el Centro Diurno del Hospital Chacón. Aquí, el horario de atención se reduce a siete horas diarias, pues la rehabilitación psicosocial es el principal foco de la institución. Desde muy temprano, los usuarios esperan en la puerta para ingresar al Centro, deseosos de comenzar. Una vez ingresados y con sus pertenencias a salvo en casilleros individuales, el golpeteo de las pelotas de ping-pong se hace presente durante las primeras horas de la mañana, donde los usuarios se divierten, practican y esperan su turno para jugar. A través del juego, los pacientes estimulan sus facultades psicomotoras mediante la lúdica, el disfrute y el compañerismo. 
Seguidamente, los estudiantes a cargo del TCU se preparan para brindar una hora de actividades educativas. Entre temas sencillos e importantes como las efemérides, las provincias de Costa Rica y la anexión del Partido de Nicoya, fue posible la socialización y construcción de conocimientos compartidos en el grupo. La elaboración propia de materiales, en la pintura de mapas y decoraciones, permitía al paciente a su vez, visualizar el propio aporte en todas aquellas actividades que se realizaban.

Transcurrido ese tiempo, llega el momento de merendar una fruta y continuar con el itinerario usual de los martes. Con paciencia, la coordinadora de Terapia Ocupacional continúa el día asignando a cada técnico un grupo para ejercitar la lecto-escritura y la lógica-matemática, capacidades necesarias en el dominio de la vida cotidiana. Desde sumar, restar, multiplicar, leer en voz alta, hasta comprender aspectos básicos de un problema o cuento, los usuarios pueden dedicar un tiempo semanal a la práctica de estas facultades con el apoyo de personal capacitado, cuyo fin último es la aplicabilidad de lo aprendido en el desenvolvimiento de la cotidianidad.

Hacia las once de la mañana, los usuarios guardan sus materiales para dirigirse hacia el salón comunitario en compañía de los estudiantes y algún encargado. Las clases son aportadas voluntariamente por una profesora de baile, que con esmero prepara la música y ciertos pasos para que los usuarios practiquen individualmente y en parejas. Aquí, la promoción de la salud desde una perspectiva integral involucra la sana dispersión, actividad física y la convivencia con otros y otras, donde los estudiantes se encargan también de involucrar a todos con su participación en la actividad. El disfrute es apreciable por los presentes, gracias a las redes de apoyo que contribuyen en el proyecto; el préstamo de instalaciones por parte de la municipalidad, el voluntariado de la profesora, el apoyo universitario y la movilización del Centro para su realización.

Después del almuerzo, los usuarios continúan desempeñando diversos roles en los programas de terapia ocupacional, llámese reciclaje, huerta, costura o computación, donde adquieren diferentes habilidades para el desempeño de ocupaciones reales. Ya sea en la venta de material reutilizado o en tejido de bufandas, cada persona puede explotar los conocimientos adquiridos para tener ganancias económicas a partir de sus esfuerzos. En un marco social donde el trabajo proporciona el sustento necesario para vivir, los proyectos 
Wimblu, Rev. Estud. de Psic. y Cienc. Soc. UCR, 14(1) 2019 (Enero-Junio): 139-152 /ISSN: 1659-2107

descritos cobran vital significancia, tanto desde un punto de vista de sobrevivencia como de realización personal.

En el recorrido de instituciones favorecidas, es necesario recordar también las labores realizadas en la Unidad de Cuidados Paliativos (UCP) de San Isidro de Heredia, ubicada en el barrio San Martín. Como ONG, la UCP brinda atención integral sin fines de lucro a personas adultas mayores en medicina general y oncológica, psicología, enfermería, nutrición, terapia física y terapia ocupacional, siendo esta última el área con la cual se aportó desde el TCU. Durante las tardes de viernes, los adultos mayores llegan desde temprano para saludar y conversar entre sí. Al tomar asiento, esperan dar por iniciadas las actividades que los estudiantes prepararon a lo largo de la semana; talleres educativos de jardinería, autocuidado, alimentación saludable, los sentidos, el aparato digestivo, y actividades de estimulación cognitiva (memoria, pensamiento lógico, psicomotricidad) y expresión emocional fueron temas que tomaron partida en el aporte universitario. Tanto desde el aporte llevado a cabo por parte del equipo del TCU como desde las labores que realizan los funcionarios de la UCP, es posible visualizar en las reuniones de los viernes el disfrute de los usuarios en actividades que promocionan su salud de una manera integral. Desde la psicoeducación hasta la estimulación, cada adulto mayor adquiere recursos muy valiosos en la convivencia con otras personas, fortaleciendo incluso de esta manera sus redes de apoyo con pares y otros profesionales que velan por su bienestar. Cerca de las tres de la tarde, la hora del café no se hace esperar, marcada por conversaciones gratas y mucho humor. Cada adulto mayor regresa a su hogar independientemente, con vivencias diferentes a lo que acostumbra en su cotidianidad.

Dentro de la literatura actualizada, se propone que la promoción de la salud orientada a la tercera edad es de suma relevancia para alcanzar un envejecimiento exitoso, caracterizado por la presencia de baja enfermedad y discapacidad, altos niveles de función cognitiva y física y un alto compromiso social. Esta última variable muestra una gran relevancia en la literatura, pues se ha encontrado que el apoyo social se asocia directamente con las habilidades cognitivas y la satisfacción de vida global (Brigeiro 2005; Morack et al. 2013; Gow et al. 2007; Vivaldi y Barra 2012). Más aún, la evidencia apunta que las personas con mayor salud integral son aquellas que establecen relaciones sociales sólidas durante su vida (Malone et al. 2016; Waldinger \& Schulz 2010; Waldinger et al. 2014), 
aspecto que se refuerza constantemente en los adultos mayores de la UCP entre personas de su misma comunidad y matrimonios que participan activamente.

Más allá de la Gran Área Metropolitana, también fue posible hacer enlace con otra comunidad interesada por la salud de sus adultos mayores. En un viaje de cinco horas, a 170 kilómetros del centro de San José, se encuentra entre un paisaje de montañas alajuelenses El Porvenir de Aguas Claras en Upala. Familiares, vecinos y encargados de la Asociación de Personas Adultas Mayores reúnen a sus parientes en tardes de sábado para realizar distintas actividades, en fomento de la convivencia y la estimulación psicosocial. Si bien se visitó una única vez a esta población por ser el primer contacto, dentro de los alcances del trabajo realizado fue posible brindar psicoeducación a los encargados sobre temáticas como el síndrome demencial y estimulación cognitiva, y tareas de estimulación musical y de memoria a los usuarios por parte del área de psicología. La cobertura de estilos de vida saludable, cuidados al paciente y salud bucodental estuvo a cargo de estudiantes de enfermería y odontología, con la atención de preguntas a cada adulto mayor que lo requiriera. Toda una tarde de vinculación y relatos bastan para comprender que la salud puede ser potenciada desde aportes breves en el trabajo con cuidadores, y en el merecimiento de que, como personas adultas mayores, tengan una escucha respetuosa y valorada de sus narraciones, ricas en experiencia y sabiduría.

De vuelta a San José, a 64 km sureste de la capital se encuentra Santa María de Dota, una zona caracterizada por los lugareños como un lugar de exuberante vegetación. Las singulares montañas rodean un asentamiento donde la problemática del suicidio se muestra cada vez más preocupante para el trabajo en salud pública. De acuerdo con la Rectoría de la Universidad de Costa Rica, datos del Ministerio de Salud revelan que la zona de los Santos conformada por "los cantones de Tarrazú, León Cortés y Dota es la zona que presenta mayor cantidad de intentos de suicidio por cada 100 mil habitantes en el país" (2014:1), con Dota en el tercer puesto de incidencia.

Toldos y mesas reunidos en la cercanía de la plaza central fueron los puestos donde múltiples disciplinas de diferentes centros ofrecieron atención e información, a través del diálogo, afiches, brochures y actividades deportivas, y la participación de diferentes grupos de TCU a lo largo del día vislumbró la importancia del aporte que brinda la acción social a poblaciones con necesidades específicas. Desde la promoción de la salud mental, fue 
Wimblu, Rev. Estud. de Psic. y Cienc. Soc. UCR, 14(1) 2019 (Enero-Junio): 139-152 /ISSN: 1659-2107

posible brindar psicoeducación de estilos de vida saludable, tales como alimentación balanceada, medidas ante el padecimiento de hipertensión, diabetes, rinitis alérgica, dislipidemias, y recomendaciones para el manejo adecuado del estrés y la ansiedad a través de técnicas como la respiración diafragmática y la detención del pensamiento. El espacio de conversación sobre dichos temas reveló precisamente la necesidad que presentan muchas personas por recibir espacios de contención, apoyo e información. Las experiencias de campo continúan reforzando que la intervención corresponde un paso de abordaje importante pero no único; el trabajo preventivo y comunitario corresponde el primer orden de atención a las problemáticas psicosociales (Brannon, Feist \& Updegraff 2013; Dickinson 2011; Sarafino \& Smith 2010; Stephens 2008). De esta manera, las ferias de salud como recursos de alcance comunitario se continúan presentando como un medio fundamental para el trabajo local en salud integral.

$* * *$

\section{Conclusiones}

Costa Rica, como un país ejemplar por su amplio sistema de salud evidencia aún la permanencia de distintas problemáticas relacionadas con salud mental. A raíz del trabajo comunal universitario fue posible llevar a cabo distintos abordajes en la materia, desde el trabajo con usuarios del Hospital Psiquiátrico Chacón Paut, hasta lugares con otro tipo de demandas como la Asociación de Personas Adultas Mayores y la Unidad de Cuidados Paliativos donde el foco poblacional es la adultez mayor. Pobladores de Santa María de Dota también ha sido centro de recepción de apoyo por parte de la acción social universitaria, en cuyo caso presente fue el aporte preventivo a través de distintas actividades informativas y recreativas.

A modo de conclusión, es importante rescatar varios aspectos que atravesaron el susodicho trabajo de campo. En primer lugar, la importancia de fomentar redes de apoyo significa para la salud mental uno de los factores de protección más importantes, lo cual puede llevarse a cabo de manera formal e informal. Desde el trabajo comunal universitario, el apoyo formal aporta con el saber interdisciplinario de diferentes disciplinas en un trabajo conjunto con las instancias comunales destinadas a la atención sanitaria. El apoyo informal 
Wimblu, Rev. Estud. de Psic. y Cienc. Soc. UCR, 14(1) 2019 (Enero-Junio): 139-152 /ISSN: 1659-2107

fue posible de observar en todas aquellas redes que de alguna u otra forma refuerzan las personas entre sí el sostén colectivo, tales como familiares, parejas, amigos, compañeros, vecinos y voluntarios.

En segundo lugar, se concluye la importancia que representa diseñar las actividades en un marco de planificación adecuado. Así, desde el planteamiento de objetivos y la secuenciación del desarrollo se contemplan las características poblaciones para que las actividades puedan ser adecuadas a la edad y a las condiciones psiquiátricas particulares de las personas.

En tercer lugar, se concluye la amplia demanda existente en el trabajo de salud mental costarricense. Desde el TCU, fue palpable la gran necesidad que expresan muchas personas de diferentes zonas nacionales en esta área de la salud, para lo cual fue limitante el recurso humano disponible. Dentro del trabajo de campo, fue particularmente visible la necesidad de trabajar el autocuidado de personas cuidadoras, especialmente aquellos que se encargan de atender personas adultas mayores y personas con deterioro cognitivo y/o psiquiátrico. Dado que el objetivo del trabajo comunal estuvo orientado a la intervención de usuarios y por la limitante del factor tiempo, no fue posible dar abordaje a esta necesidad, entre muchas otras que distintas personas expresaban de forma individual.

Ante esto, cabe destacar la importancia de que, como profesionales, nuestro enfoque se oriente en miras de una salud integral que integre los tres ejes bio-psico-social. Con un énfasis radical en la atención de primer orden, la prevención y promoción de la salud pueden aportar a la sociedad costarricense un aumento en la calidad de vida de las personas, para lo cual es imprescindible hacer un llamado a las diferentes instancias que trabajan en la materia. Un trabajo interdisciplinario e intersectorial que actúe de la mano con los aportes de la acción social universitaria, abrirá puentes para construir una sociedad con mayor bienestar.

\section{Bibliografía}

Ardila, Alfredo y Mónica Rosselli. 2007. Neuropsicología Clínica. México D.F.: Editorial El Manual Moderno. 
Wimblu, Rev. Estud. de Psic. y Cienc. Soc. UCR, 14(1) 2019 (Enero-Junio): 139-152 /ISSN: 1659-2107

Brannon, Linda, Jess Feist \& John Updegraff. 2013. Health Psychology. An Introduction to Behavior and Health. Belmont: Cengage Learning.

Brigeiro, Mauro. 2005. "Envejecimiento exitoso" y "tercera edad": Problemas y retos para la promoción de la salud. Investigación y Educación en Enfermería. XXIII (1): 102109. Medellín: Universidad de Antioquia.

Caja Costarricense del Seguro Social. 2017. Anuario estadístico 2017. San José: Costa Rica. https://www.ccss.sa.cr/est_salud. Consulta: 15 de diciembre de 2018.

Consejo Universitario. 2018. Reglamento del Trabajo Comunal Universitario. Gaceta Universitaria, 18-2018. San José: Unidad de Información de la Universidad de Costa Rica. http://www.cu.ucr.ac.cr/normativ/trabajo_comunal.pdf. Consulta: 15 de diciembre de 2018.

Dickinson, Emily. 2011. “Community Approaches Within Health Psychology”. En: Marks, David, Murray, Michael, Evans, Brian, \& Vida-Estacio, Emee. 2011. Health Psychology: Theory, research and practice, Chapter 15: 338-364. London: SAGE Publications, Ltd.

Gow, Alan et al. 2007. "Social Support and Successful Aging. Investigating the Relationship Between Lifetime Cognitive Change and Life Satisfaction”. Journal of Individual Differences. XXVIII (3): 103-115. DOI: 10.1027/1614-0001.28.3.103

IESM-OMS. 2008. Informe sobre el sistema de salud mental en Costa Rica. San José: Ministerio de Salud de Costa Rica. http:/www.bvs.sa.cr/saludmental/informe.pdf. Consulta: 15 de diciembre de 2018.

Jara, Marisol. 2018. Información general del proyecto Estrategias para la promoción del mejoramiento de la salud mental. San José: Oficina de Planificación Universitaria y Sistema Institucional Plan-Presupuesto de la Universidad de Costa Rica.

Labrador, Francisco. 2009. Técnicas de Modificación de Conducta. Madrid: Ediciones Pirámide.

Lezak et al. 2012. Neuropsychological Assessment (5th. Ed.). New York: Oxford University Press.

Malone et al. 2016. "Midlife Eriksonian Psychosocial Development: Setting the Stage for Late-Life Cognitive and Emotional Health”. Developmental Psychology. LII. USA: Hardvard Study of Adult Development. DOI: 10.1037/a0039875. 
Wimblu, Rev. Estud. de Psic. y Cienc. Soc. UCR, 14(1) 2019 (Enero-Junio): 139-152 /ISSN: 1659-2107

Ministerio de Salud. 2012. Política Nacional de Salud Mental 2012-2021. San José, Costa Rica: Producción Documental del Ministerio de Salud. http://www.bvs.sa.cr/saludmental/politicasaludmental.pdf. Consulta: 15 de diciembre de 2018.

Moore, McKay \& Catherine Mateer. 2001. Cognitive Rehabilitation. An Integrative Neuropsychological Approach. New York: The Guilford Press.

Morack, Jennifer et al. 2013. "Multidomain Trajectories of Psychological Functioning in Old Age: A Longitudinal Pespective on (Uneven) Successful Aging”. Developmental Psychology. XLIX (12): 2309-2324. DOI: 10.1037/a0032267

Organización Mundial de la Salud. 2013. Salud mental: Un estado de bienestar. https://www.who.int/features/factfiles/mental_health/es/. Consulta: 15 de diciembre de 2018.

Organización para la Cooperación y el Desarrollo Económico. 2017. Estudios de la OCDE sobre los sistemas de salud: Costa Rica. Evaluación y recomendaciones. San José: Ministerio de Salud. https://www.ministeriodesalud.go.cr/index.php/biblioteca-dearchivos/tramites/registros-de-gestores-de-la-salud/ocde-residuos/3564-estudios-dela-ocde-sobre-los-sistemas-de-salud-costa-rica/file. Consulta: 15 de diciembre de 2018.

Portellano, José. 2005. Introducción a la Neuropsicología. Madrid: McGraw-Hill.

Presidencia de la República de Costa Rica. 2016. CCSS alcanza cobertura de 1.041 Ebáis en todo el país. https://presidencia.go.cr/sin-categoria/2016/06/ccss-alcanzacobertura-de-1-041-ebais-en-todo-el-pais/. Consulta: 15 de diciembre de 2018.

Rectoría de la Universidad de Costa Rica. 2014. UCR investiga tendencias de suicidios en habitantes de

Dota. http://www.rectoria.ucr.ac.cr/site/wp-content/uploads/2014/11/columna-rectoria-1911-14corregido3.pdf3. Consulta: 15 de diciembre de 2018.

Sarafino, Edward \& Timothy Smith. 2010. Health Psychology: Biopsychosocial Interactions. USA: John Wiley \& Sons, Inc.

Stephens, Christine. 2008. Health promotion. A psychosocial approach. New York: McGraw Hill. 
Wimblu, Rev. Estud. de Psic. y Cienc. Soc. UCR, 14(1) 2019 (Enero-Junio): 139-152 /ISSN: 1659-2107

Vivaldi, Flavia y Enrique Barra. 2012. “Bienestar Psicológico, Apoyo Social Percibido y Percepción de Salud en Adultos Mayores”. Terapia Psicológica, XXX (2): 23-29. Concepción: Sociedad Chilena de Psicología Clínica.

Waldinger, Robert \& Marc Schulz. 2010. "What's love got to do with it? Social function, perceived health, and daily happiness in married octogenarians". Psychology and Aging. XXV (2): 422-31. USA: Hardvard Study of Adult Development. DOI: $10.1037 / \mathrm{a} 0019087\}$

Waldinger, Robert et al. 2014. "Security of Attachment to Spouses in Late Life: Concurrent and Prospective Links With Cognitive and Emotional Well-Being”. Clinical Psychological Science. III. USA: Hardvard Study of Adult Development. DOI: $10.1177 / 2167702614541261$

Wilson, Barbara et al. 2017. Neuropsychological Rehabilitation. The International Handbook. New York: Taylor \& Francis Group.

Wood, Stephen, Nicholas Allen \& Christos Pantelis. 2009. The Neuropsychology of Mental Illness. New York: Cambridge University Press. 